\title{
Comparisons of tooth size and morphology between the late Miocene hominoids from Lufeng and Yuanmou, China, and their implications
}

\author{
WU LIU ${ }^{1 *}$, LIANG ZHENG ${ }^{2}$ \\ ${ }^{1}$ Institute of Vertebrate Paleontology and Paleoanthropology, Chinese Academy of Sciences, Beijing 100044, China \\ ${ }^{2}$ Yunnan Provincial Institute of Archaeology, Kunming, China
}

Received 30 June 2003; accepted 30 March 2004

\begin{abstract}
The relationship between the Yuanmou hominoid and the Lufeng hominoid (Lufengpithecus lufengensis), both from Yunnan Province, China, and among the most abundantly represented fossil hominoid of Eurasia, has been the subject of much debate. In the past 10 years, comparative studies of cranial and dental morphology of the Lufeng and Yuanmou hominoids indicate that the two hominoids resemble each other more than either of them resemble any other Miocene hominoid. In this paper, we summarize the dental differences seen between the Yuanmou and Lufeng hominoids, and discuss their implications. Our results show that the Lufeng hominoid has relatively smaller front teeth, smaller $\mathrm{M}^{1}$, and higher SQ than the Yuanmou hominoid, indicating a more folivorous or soft diet, such as leaves and berries, in the former. Tooth wear analysis indicates that both upper and lower molars of the Yuanmou hominoid were more heavily worn than those of the Lufeng hominoid, offering additional support to the findings of tooth size proportion and shearing crest development. We propose that different diets might have characterized the two hominoid populations, the differences possibly related to the environment, behavior pattern, and population structure of the Yuanmou and Lufeng hominoids.
\end{abstract}

Key words: Yuanmou, Lufeng, hominoids, Lufengpithecus, teeth and diet

\section{Introduction}

Late Miocene hominoids found at the sites of Lufeng and Yuanmou of Yunnan Province in southwestern China are among the most abundantly known fossil hominoids of Eurasia. The two sites are very close to each other in both geographical distance and geological age (He, 1997). Comparative studies of cranial and dental morphology conducted in the past decade, including metric and morphometric analyses of the Yuanmou and Lufeng hominoids, indicate that the two hominoid assemblages resemble each other more than either of them resemble any other Miocene hominoid from elsewhere in the world (Zheng and Zhang, 1997; Liu et al., 2000, 2001a, b). However, there are still some obvious differences between the Yuanmou and Lufeng hominoids, and it has been proposed that that the two should be put into the same genus with species-level differences, i.e. Lufengpithecus lufengensis for the Lufeng and L. hudienensis (or L. keiyuanensis) for the Yuanmou hominoid (Zheng and Zhang, 1997; Harrison et al., 2002; Schwartz et al., 2003).

Recently, a series of studies has focused on a comparison of tooth size, morphology, wear pattern, and microstructure of the Yuanmou and Lufeng hominoid fossils (Liu et al., 2000, 2002; Schwartz et al., 2003). Through these studies

* Corresponding author. e-mail: liuwu@ivpp.ac.cn phone: +86-10-68935120; fax: +86-10-68337001

Published online 11 August 2004

in J-STAGE (www.jstage.jst.go.jp) DOI: 10.1537/ase.04S011 we found some obvious metric, morphological, microstructural and wear differences between teeth of the two hominoid assemblages. Most of the differences seem to be related to diet, behavior, population structure, and probably environmental differences between the two hominoids. In the present study, we briefly summarize differences between the Yuanmou and Lufeng hominoids revealed by the recent studies, and discuss the implications of these differences in interpreting the relationship between the two.

Except for the analysis of tooth size, all dental features discussed here are those concerning molars. Nearly all the molars are isolated teeth. The determination of the first, second, and third molars was made in part on the basis of the interstitial wear facets. Because the mesial side of the first molars is in contact with the second deciduous molar and, later, the permanent fourth premolar, the mesial side of the first molar typically has two wear facets. Second molars have only one proximal wear facet. Third molars also have only one proximal wear facet and lack a distal facet (Liu et al., 2001a). The details of the materials and methods used in each of the studies referred to can be found in the respective references (Liu et al., 2000, 2002; Schwartz et al., 2003).

\section{Tooth Size}

The mesiodistal (MD) and buccolingual (BL) tooth crown dimensions of the of the Yuanmou hominoid were measured and compared with those of the Lufeng hominoid (Table 1, Table 2). Figure 1 shows calculated crown areas of the upper 
Table 1. Basic statistics of Yuanmou hominoid tooth dimensions

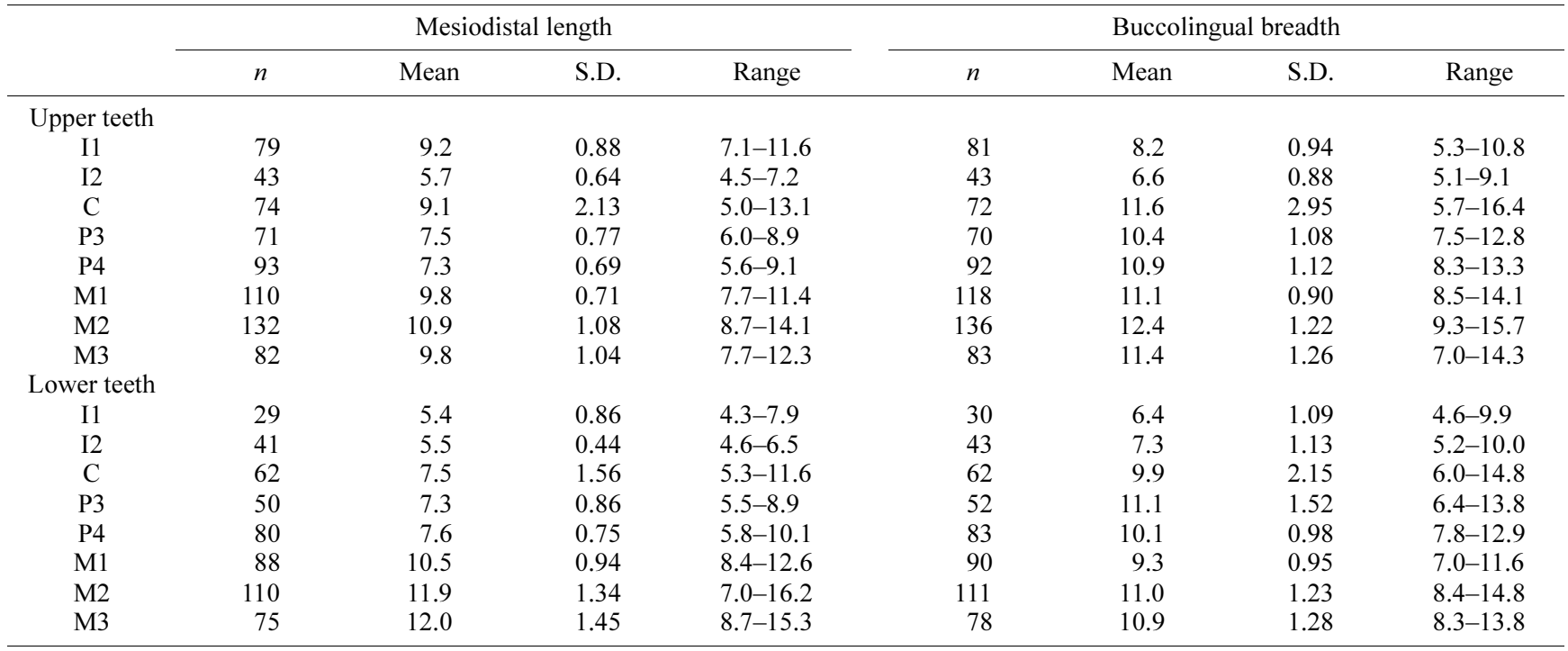

Values are in $\mathrm{mm}$.

Table 2. Basic statistics of Lufeng hominoid tooth dimensions

\begin{tabular}{|c|c|c|c|c|c|c|c|c|}
\hline & \multicolumn{4}{|c|}{ Mesiodistal length } & \multicolumn{4}{|c|}{ Buccolingual breadth } \\
\hline & $n$ & Mean & S.D. & Range & $n$ & Mean & S.D. & Range \\
\hline \multicolumn{9}{|c|}{ Upper teeth } \\
\hline $\mathrm{I} 1$ & 47 & 9.9 & 0.8 & $8.3-11.4$ & 62 & 8.9 & 0.8 & $7.5-11.0$ \\
\hline $\mathrm{I} 2$ & 30 & 6.0 & 0.6 & $4.8-7.3$ & 30 & 6.8 & 0.7 & $5.7-8.5$ \\
\hline $\mathrm{C}$ & 32 & 11.5 & 2.0 & $7.5-16.8$ & 32 & 10.4 & 1.9 & $6.4-17.3$ \\
\hline P3 & 34 & 8.4 & 1.0 & $6.9-10.1$ & 35 & 11.4 & 1.3 & $9.3-13.4$ \\
\hline $\mathrm{P} 4$ & 38 & 7.7 & 0.7 & $6.5-8.8$ & 38 & 11.6 & 1.1 & $9.8-13.6$ \\
\hline M1 & 41 & 11.1 & 1.0 & $9.8-13.5$ & 41 & 12.1 & 1.1 & $10.5-14.8$ \\
\hline M2 & 44 & 12.7 & 1.3 & $10.4-15.6$ & 44 & 13.7 & 1.3 & $11.3-16.6$ \\
\hline M3 & 24 & 12.1 & 1.5 & $10.1-14.2$ & 23 & 13.3 & 1.5 & $10.7-15.3$ \\
\hline \multicolumn{9}{|c|}{ Lower teeth } \\
\hline I1 & 33 & 5.6 & 0.5 & $4.9-7.0$ & 34 & 7.6 & 0.9 & $6.1-9.9$ \\
\hline $\mathrm{I} 2$ & 35 & 5.9 & 0.5 & $5.0-7.0$ & 35 & 8.6 & 1.2 & $7.1-12.2$ \\
\hline $\mathrm{C}$ & 48 & 7.5 & 1.3 & $5.9-10.5$ & 47 & 10.4 & 1.6 & $8.4-15.0$ \\
\hline P3 & 32 & 9.2 & 1.4 & $7.5-13.0$ & 33 & 10.7 & 1.6 & $8.6-13.3$ \\
\hline $\mathrm{P} 4$ & 47 & 9.0 & 1.1 & $6.5-11.0$ & 46 & 9.7 & 0.9 & $8.3-11.6$ \\
\hline M1 & 42 & 11.7 & 1.1 & $10.0-14.2$ & 42 & 10.3 & 1.1 & $8.8-12.4$ \\
\hline M2 & 62 & 13.4 & 1.4 & $10.1-15.5$ & 59 & 12.0 & 1.3 & $9.5-14.4$ \\
\hline M3 & 38 & 13.6 & 1.3 & $11.5-16.7$ & 39 & 11.7 & 1.2 & $10.1-14.6$ \\
\hline
\end{tabular}

Values are in mm, cited from Wood and Xu (1991).

and lower teeth, comparing the Yuanmou, Lufeng, and Kaiyuan assemblages. Results show that the Lufeng hominoid exceeds the Yuanmou hominoid in size of all teeth. Figure 1 also indicates that among the three hominoid fossil assemblages found in Yunnan, tooth size is more comparable between the Yuanmou and Kaiyuan assemblages.

Previous studies have demonstrated that diets of fossil hominoids are related to tooth size and that species with larger incisors tend to consume larger, tougher fruits, whereas those with smaller front teeth tend to feed on smaller-sized foods or those that require less extensive incisal preparation, such as leaves or berries. The ratio of the M1 and M3 areas also shows a pattern in which a high M1 to
M3 ratio indicates consumption of relatively more fruits (Teaford and Ungar, 2000). We calculated the above two ratios for the Yuanmou and Lufeng hominoids, and showed that in both $\mathrm{I}^{1} / \mathrm{M}^{1}$ MD lengths $(0.94$ and 0.89 , in Yuanmou and Lufeng, respectively) and $\mathrm{M}^{1} / \mathrm{M}^{3}$ areas (0.96 and 0.84 , respectively) the Yuanmou hominoid exhibits larger values than the Lufeng hominoid (Liu et al., 2002).

\section{Shearing Crest Development of Lower Molars}

Given the close relationship between diet and development of the shearing crests of molars (Teaford and Ungar, 2000), we observed and measured the shearing crests on the 

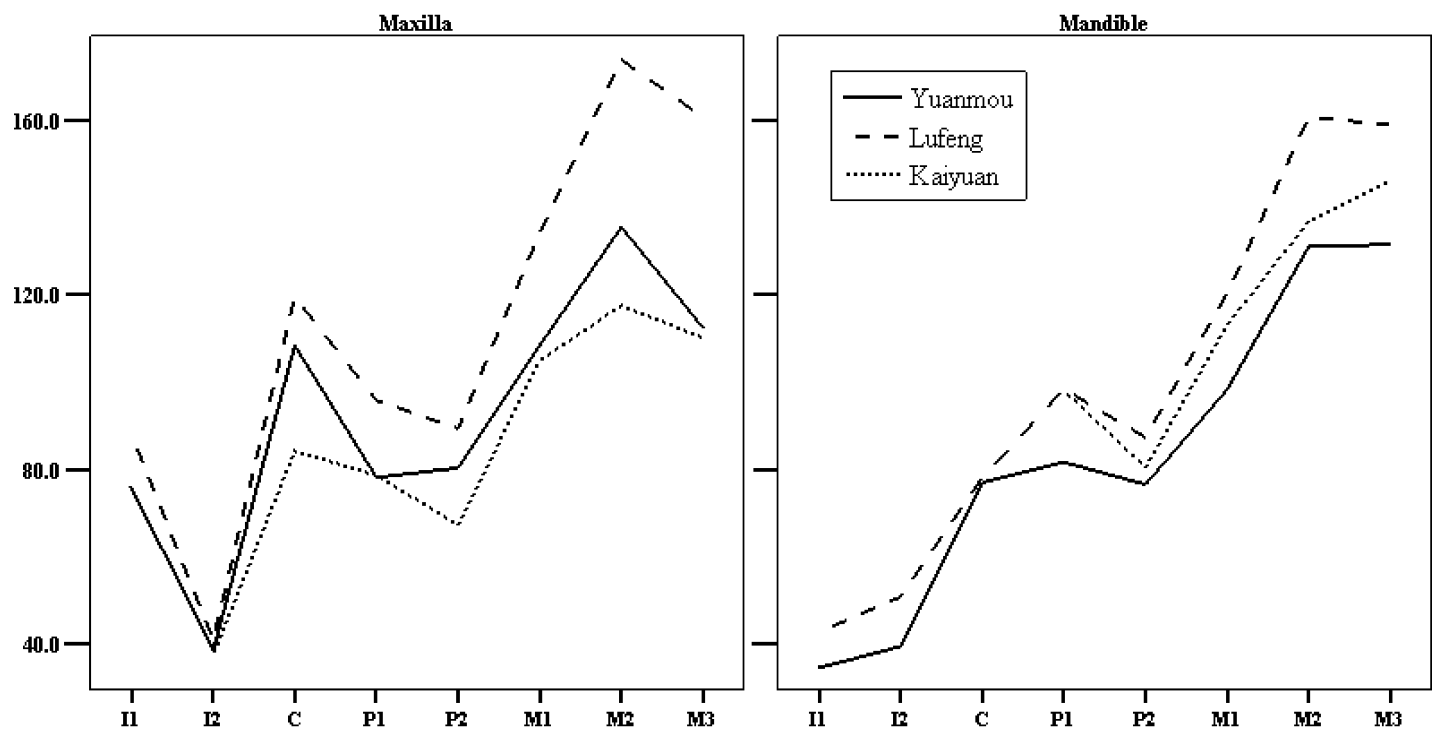

Figure 1. Calculated tooth crown areas of the upper and lower teeth of the Yuanmou and Lufeng hominoids.

lower second molars of both the Yuanmou and Lufeng hominoids; the shearing quotient (SQ) was calculated as an indicator of shearing crest development (e.g. Kay, 1984; Kay and Covert, 1984; Kay and Ungar, 1997). Our analysis showed that the SQ values of the Yuanmou and Lufeng molars are -13.4 and -4.7 , respectively, indicating that the former has less developed shearing crests than the latter, and that more folivorous food components like leaves and berries were probably consumed by the Lufeng than by the Yuanmou hominoid (Liu et al., 2002). This result fits with the findings of the $\mathrm{I}^{1} / \mathrm{M}^{1}$ length and $\mathrm{M}^{1} / \mathrm{M}^{3}$ area indices.

\section{Tooth Wear Differences}

During field excavation and laboratory analysis we noticed that there are very obvious differences in tooth wear patterns between the two hominoid assemblages. The molars of the Yuanmou hominoid are usually more heavily worn than those of the Lufeng assemblage. Given that there is possibly a close relationship between tooth wear and diet, behavior pattern, environment, and population structure, we set a three-grade standard (Figure 2) to compare molar wear between the two hominoid assemblages. Tooth wear of the Yuanmou and Lufeng hominoids evaluated by means of the current scoring standard shows that both the upper and lower molars from Yuanmou tend to be more heavily worn than those from Lufeng (Table 3, Figure 3).

\section{Enamel Thickness}

Recently, a histological analysis of two lower molars of the Yuanmou hominoid was carried out and the results were compared with corresponding data of the Lufeng specimens (Schwartz et al., 2003). That study revealed several obvious differences in dental microstructure between the two hominoids, including enamel secretion rate, crown formation time, striae morphology, and enamel thickness. Among these features, enamel thickness exhibited the most obvious difference. Relative enamel thickness in one Yuanmou molar was 14.1, which is much thinner than the same measure reported for Lufeng specimens (average of 24.2).

\section{Discussion}

In the present study, we concentrated on the dental differences observed between the Yuanmou and Lufeng hominoids. We believe that the main differences between the two can be expressed by four aspects: tooth size, shearing crest development of lower molars, tooth wear, and enamel thickness. Obvious differences were found in all of these four features. With regard to tooth size, the MD and BL crown dimensions of all upper and lower teeth of the Lufeng hominoid are larger than those of the Yuanmou hominoid. In tooth size proportion, the Lufeng hominoid has relatively smaller $\mathrm{I}^{1} / \mathrm{M}^{1}$ and $\mathrm{M}^{1} / \mathrm{M}^{3}$ ratios than the Yuanmou hominoid. In development of lower molar shearing crests, the Yuanmou hominoid molar has a much lower SQ value, indicating less developed shearing crests than in that of the Lufeng hominoid. The third feature, which seems to be more interesting,

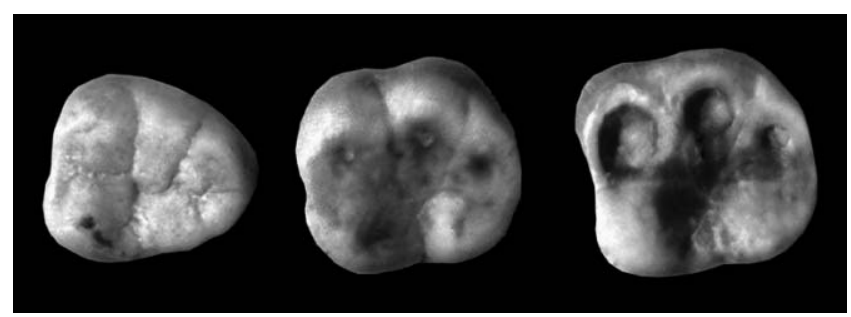

Figure 2. Tooth wear grades. Left: grade 1, no wear or slight wear on cusps without dentine exposure; middle: grade 2 , moderate tooth wear on cusps with point dentine exposures; right: grade 3 , tooth cusps are worn out and dentine exposures occupy a large area of the occlusal surface. 
Table 3. Molar wear of the Yuanmou and Lufeng hominoids

\begin{tabular}{|c|c|c|c|c|c|c|c|c|c|c|c|c|c|c|c|c|}
\hline \multirow{2}{*}{$\begin{array}{l}\text { Tooth type } \\
\text { Wear grade }\end{array}$} & & \multicolumn{3}{|c|}{ M1 } & \multicolumn{3}{|c|}{ M2 } & \multicolumn{3}{|c|}{ M3 } & \multicolumn{3}{|c|}{ M1 or M2 } & \multicolumn{3}{|c|}{ Pooled } \\
\hline & & 1 & 2 & 3 & 1 & 2 & 3 & 1 & 2 & 3 & 1 & 2 & 3 & 1 & 2 & 3 \\
\hline \multicolumn{17}{|c|}{ Upper Molars } \\
\hline \multirow[t]{2}{*}{ Yuanmou } & $n$ & 43 & 44 & 32 & 43 & 37 & 32 & 38 & 23 & 18 & 26 & 27 & 18 & 150 & 131 & 100 \\
\hline & $\%$ & 36.1 & 37.0 & 26.9 & 38.4 & 33.0 & 28.6 & 48.1 & 29.1 & 32.9 & 36.6 & 38.0 & 25.3 & 39.4 & 34.4 & 26.2 \\
\hline \multirow[t]{2}{*}{ Lufeng } & $n$ & 11 & 4 & 1 & 10 & 5 & 0 & 9 & 3 & 1 & & & & 30 & 12 & 2 \\
\hline & $\%$ & 68.7 & 25.0 & 6.3 & 66.7 & 33.3 & 0.0 & 69.2 & 23.1 & 7.7 & & & & 68.1 & 27.3 & 4.6 \\
\hline \multicolumn{17}{|c|}{ Lower Molars } \\
\hline \multirow[t]{2}{*}{ Yuanmou } & $n$ & 26 & 26 & 22 & 34 & 23 & 40 & 38 & 22 & 16 & 33 & 22 & 22 & 131 & 93 & 100 \\
\hline & $\%$ & 35.1 & 35.1 & 29.7 & 35.1 & 23.7 & 41.2 & 50.0 & 29.0 & 21.0 & 42.9 & 28.6 & 28.6 & 40.4 & 28.7 & 30.9 \\
\hline \multirow[t]{2}{*}{ Lufeng } & $n$ & 10 & 6 & 3 & 16 & 2 & 0 & 8 & 3 & 2 & 5 & 0 & 0 & 39 & 11 & 5 \\
\hline & $\%$ & 52.6 & 31.6 & 15.8 & 88.9 & 11.1 & 0.0 & 61.5 & 23.1 & 15.4 & 100.0 & 0.0 & 0.0 & 70.9 & 20.0 & 9.1 \\
\hline
\end{tabular}
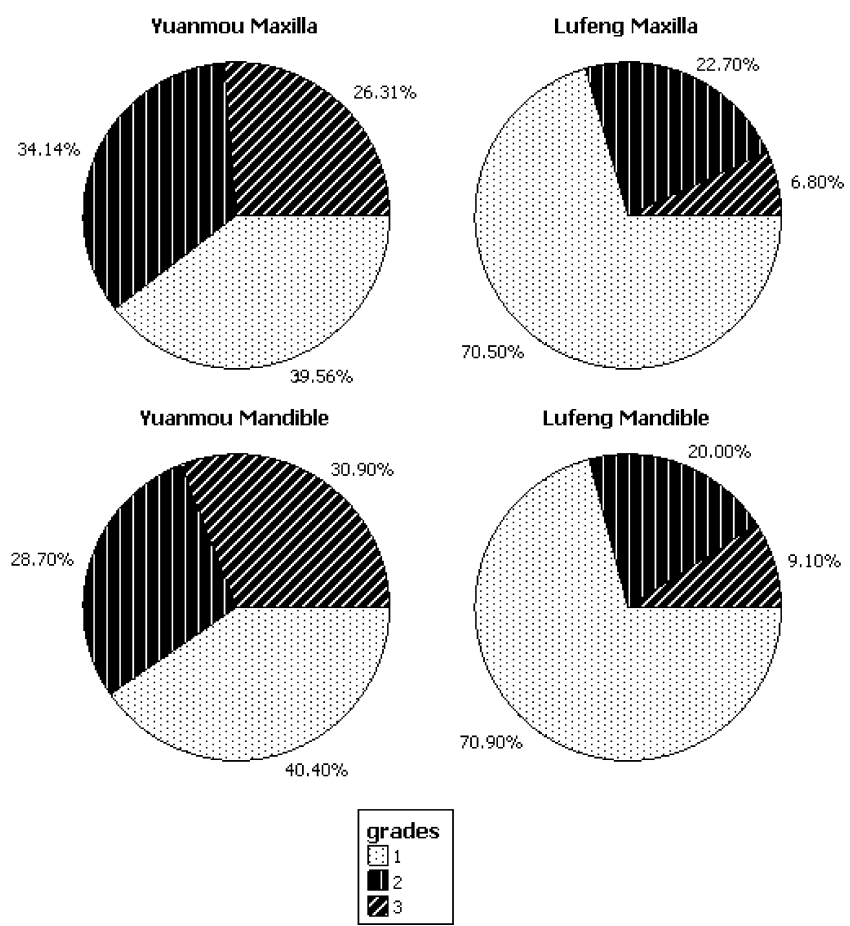

Figure 3. Tooth wear comparison of the pooled upper and lower molar assemblages of the Yuanmou and Lufeng hominoid.

shows much heavier wear to occur in both the upper and lower molars of the Yuanmou than in the same teeth of the Lufeng hominoid. With regards to structural information obtained from histological analysis, enamel thickness of one Yuanmou hominoid lower molar was found to be much thinner than the average Lufeng condition.

Referring to the previous studies by others (Andrews and Martin, 1991; Hylander, 1975; Teaford and Ungar, 2000), all four features discussed above can be related to food texture, and also, to lesser extents, to feeding behavior, population structure, and environment. With tooth size proportion and shearing crest development, those hominoids with higher $\mathrm{I}^{1} /$ $\mathrm{M}^{1}$ and $\mathrm{M}^{1} / \mathrm{M}^{3}$ ratios and lower SQ values tend to consume harder fruits. The Yuanmou hominoid, with relatively larger front teeth and weakly developed molar shearing crests, probably subsisted on a harder or frugivorous diet, while the Lufeng hominoid favored softer foods like leaves and berries. Molar wear is considered to be closely related to the textures of the foods eaten, because the main function of molars is to chew, smash, and grind food. Therefore, the primary reason for differences seen between molar wear of the two hominoids is most likely the textures of the food consumed. We presume that relatively hard foods caused the heavier molar wear of the Yuanmou hominoid, whereas the Lufeng hominoid is thought to have fed on softer diets. This finding corresponds well with the results of tooth size proportion and shearing crest development.

As for the relationship between enamel thickness and diet, some early studies demonstrated that leaf-eating species tend to have thinner enamel than fruit-eating species (Kay, 1985). For fossil species, some authors hypothesize that the thicker enamel of australopithecines suggests that they ate very hard, brittle foods more so than do living primates (Kay, 1985; Martin, 1985). But this view has been challenged by new evidence which shows that some fossil taxa like Otavipithecus and Ardipithecus have thin enamel (White et al., 1994; Teaford and Ungar, 2000). Also, recent studies indicate that there are many potential complicating factors related to the functional significance of enamel thickness. For example, thick enamel by itself does not necessarily provide protection against hard objects, which may cause fracturing of enamel (Pilbeam, 1997; Schwartz, 2000). So, the correlation between enamel thickness and diet may not be sufficiently strong for enamel thickness to be a reliable indicator of diet. Also, enamel thickness measurements of the Yuanmou hominoid come from only one molar, which may not be representative of their enamel thickness.

Based on the above discussion, we are inclined to think that the diets of the Yuanmou and Lufeng hominoids were strikingly different from each other. The different diets of the Yuanmou and Lufeng hominoids further indicate that they may have had different food sources. If so, the fauna and flora associated with the two hominoids may not have been identical. A recent analysis of micromammals of the two hominoid sites indicates a different faunal composition between the sites ( $\mathrm{Ni}$ and Qiu, 2002). We think that the Yuanmou and Lufeng hominoids might have lived in different environments. Currently, some colleagues tend to put the hominoids from Yuanmou and Lufeng into different species 
(Harrison et al., 2002; Schwartz et al., 2003). If so, it is possible that they had different behaviors. We believe that behavior patterns such as living habits and locomotion could have lead to differences of food choice. Even if the Yuanmou and Lufeng hominoids inhabited similar environments, if their behavior patterns were different, they could also have had diets with very different food textures. Finally, tooth wear is directly related to age at death, which reflects population structure and overall health. We presume that the tooth type composition of our study represents normal population compositions for both the Yuanmou and Lufeng hominoids. We believe that even though tooth wear differences between the Yuanmou and Lufeng hominoids were caused mainly by different diets and also by different environments and behaviors, currently we cannot fully exclude the possible influences of population structure and health conditions.

We believe that except for diet, all differences mentioned in the present study may relate to possible influences of a different environment, behavior pattern, and even population structure between the Yuanmou and Lufeng hominoids. Whether or not these differences are influenced by phylogenetic affinities needs further verification.

\section{Acknowledgments}

We wish to express our sincere thanks to the colleagues from different institutions and museums for their efforts in the collection of fossils in the field and their kind help to our studies of the fossils. This work was supported by National Key Research Project of China (95-01).

\section{References}

Andrews P. and Martin L. (1991) Hominoid dietary evolution. Philosophical Transactions of the Royal Society of London, Series B, 334: 199-209.

Harrison T., Ji X., and Su D. (2002) On the systematic status of the late Neogene hominoids from Yunnan Province, China. Journal of Human Evolution, 43: 207-227.

He Z.Q. (1997) Yuanmou Hominoid Fauna. Yunnan Science Press, Kunming.

Hylander W.L. (1975) Incisor size and diet in anthropoids with special reference to Cercopithecidae. Science, 189: 10951098.

Kay R. (1984) On the use of anatomical features to infer foraging behavior in extinct primates. In: Rodman P.S. and Cant J.G.H. (eds.), Adaptation for Foraging in Nonhuman Primates: Contributions to an Organismal Biology of Prosimians, Monkeys, and Apes. Columbia University Press, New York, pp. 21-53.

Kay R. (1985) Dental evidence for the diet of Australopithecus. Annual Review of Anthropology, 14: 315-341.
Kay R. and Covert (1984) Anatomy and behavior of extinct primates. In: Chivers D.J., Wood B.A., and Bilsborough A. (eds.), Food Acquisition and Processing in Primates. Plenum Press, New York, pp. 467-508.

Kay R. and Ungar P. (1997) Dental evidence for diet in some Miocene catarrhines with comments on the effects of phylogeny on the interpretation of adaptation. In: Begun D.R., Ward C.V., and Rose M.D. (eds.), Function, Phylogeny, and Fossils: Miocene Hominoid Evolution and Adaptations. Plenum Press, New York, pp. 131-151.

Liu W., Zheng L., and Jiang C. (2000) Statistical analyses of the metric data of hominoid teeth found in Yuanmou of China. Chinese Science Bulletin, 45: 936-942.

Liu W., Hlusko L., and Zheng L. (2001a) Morphometric analysis of hominoid lower molars found in Yuanmou of Yunnan Province, China. Primates. 42: 123-134.

Liu W., Zheng L., and Walker A. (2001b) Three-dimensional morphometric analyses of hominoid lower molars from Yuanmou of Yunnan Province, China. Acta Anthropologica Sinica, 20: $163-177$.

Liu W., Gao F., and Zheng L. (2002) The diet of the Yuanmou hominoid, Yunnan province, China: an analysis from tooth size and morphology. Anthropological Science, 110: 149163.

Martin L.B. (1985) Significance of enamel thickness in hominoid evolution. Nature, 314: 260-263.

Ni X. and Qiu Z. (2002) The micromammalian fauna from the Leilao, Yuanmou hominoid locality: implications for biochronology and paleoecology. Journal of Human Evolution, 42: 535-546.

Pilbeam D.R. (1997) Research on Miocene hominoids and hominid origins: the last three decades. In: Begun D.R., Ward C.V., and Rose M.D. (eds.), Function, Phylogeny and Fossils: Miocene Hominoid Evolution and Adaptations. Plenum Press, New York, pp. 13-28.

Schwartz G.T. (2000) Taxonomic and functional aspects of the patterning of enamel thickness distribution in extant large-bodied hominoids. American Journal of Physical Anthropology, 111: $221-244$

Schwartz G., Liu W., and Zheng L. (2003) Preliminary investigation of dental microstructure in the Yuanmou hominoid (Lufengpithecus hudienensis), Yunnan Province, China. Journal of Human Evolution, 44: 189-202.

Teaford M.F. and Ungar P. (2000) Diet and the evolution of the earliest human ancestors. Proceedings of the National Academy of Sciences of the United States of America, 97: 1350613511.

White T.D., Suwa G., and Asfaw B. (1994) Australopithecus rami$d u s$, a new species of early hominid from Aramis, Ethiopia. Nature, 371: 306-312.

Wood B.A. and Xu Q. (1991) Variation in the Lufeng dental remains. Journal of Human Evolution, 20: 291-311.

Zheng L. and Zhang X.Y. (1997) Hominoid fossils. In: He Z.Q. (ed.), Yuanmou Hominoid Fauna. Yunnan Science Press, Kunming, pp. 21-58. 